\title{
TV/Series
}

$6 \mid 2014$

Écho et reprise dans les séries télévisées (III) : de la métafiction à la transmédialité

\section{Échos lointains pour reprise dissonante : la série catalane Infidels}

\section{Jennifer Houdiard}

\section{(2) OpenEdition}

\section{Journals}

Édition électronique

URL : http://journals.openedition.org/tvseries/325

DOI : $10.4000 /$ tvseries.325

ISSN : 2266-0909

Éditeur

GRIC - Groupe de recherche Identités et Cultures

Référence électronique

Jennifer Houdiard, « Échos lointains pour reprise dissonante : la série catalane Infidels », TV/Series [En ligne], 6 | 2014, mis en ligne le 01 décembre 2014, consulté le 30 avril 2019. URL : http://

journals.openedition.org/tvseries/325; DOI : 10.4000/tvseries.325

\section{(c) (i) (9)}

TV/Series est mis à disposition selon les termes de la licence Creative Commons Attribution - Pas d'Utilisation Commerciale - Pas de Modification 4.0 International. 


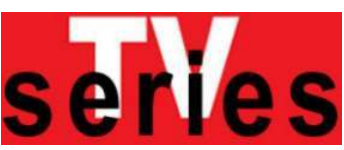

\title{
Échos lointains pour reprise dissonante : la série catalane Infidels
}

Jennifer HOUDIARD

\begin{abstract}
La série Infidels (TV3, 2009-2011), diffusée sur la chaîne catalane ainsi que sur internet durant trois saisons, était attendue comme une sorte d'adaptation de séries " féminines » américaines telles que Sex and the City (HBO, 1998-2004) ou Desperate Housewives (ABC, 2004). Cependant, une fois les premiers épisodes diffusés, Infidels fut rapidement reconnue comme une production originale, à double titre. En effet, loin de se limiter à transposer au cadre barcelonais des fictions télévisées venues des États-Unis, les scénaristes ont réussi à concevoir une fiction «maison » de qualité, fidèle à l'esprit de la chaîne TV 3 par son ancrage dans la re-création d'un contexte catalan identifiable comme réel et réaliste par le spectateur. Si des "clins d'œil » aux séries américaines citées plus haut ou à la série britannique Mistresses (BBC One, 2008-2010) sont aisément perceptibles, ils se limitent en général à un jeu sur l'intertextualité.

Au-delà de l'originalité et de la «catalanité » d'Infidels, les journalistes et critiques ont célébré le traitement profondément novateur des figures féminines et, plus généralement, la remise en question encore inédite des clichés et des rôles traditionnels dévolus aux femmes ainsi qu'aux personnages féminins qui peuplent la grande majorité des fictions télévisées. Les cinq protagonistes de la série ont en commun l'infidélité sexuelle et sentimentale, il est vrai, mais à mesure que les épisodes s'enchaînent, la quête ontologique qui sous-tend la diégèse et lui sert de fil conducteur se révèle de plus en plus clairement : Paula, Lídia, Cruz, Joana et Arlet sont, finalement, infidèles aussi bien aux rôles de genre traditionnels qu'au modèle incarné par le nouvel « Éternel féminin » des médias de masse, et ce n'est qu'ainsi qu'elles peuvent devenir fidèles à elles-mêmes.
\end{abstract}

$\mathrm{L}$ a série Infidels a été diffusée durant trois saisons, de 2009 à 2011, sur TV3 ainsi que sur Internet. Il s'agit de la première série « féminine » produite par Diagonal TV et diffusée sur la chaine publique catalane: l'action d'Infidels est centrée sur cinq protagonistes, cinq amies barcelonaises qui ne sont pas sans évoquer les héroïnes de Desperate Housewives (ABC, 2004-2012) ou de Sex and the City (HBO, 1998-2004). Cependant, ses créateurs la définissent comme une fiction originale et résolument catalane, une série " féminine, mais sur des femmes de chez nous, qui n'a rien à voir avec les séries féminines étrangères. ${ }^{1}$ » Pour Jordi Roure, directeur des programmes de fiction de TV3, Infidels est un pari, un nouveau défi pour une chaîne dont les fictions s'adressent habituellement à toute la famille, sans cibler un genre en particulier : " Pendant longtemps, nous avons été obligés de proposer des productions qui visaient à satisfaire l'ensemble de la famille. [Infidels] est une série différente, qui fait appel aux émotions et aux sentiments et met en scène des femmes qui ont décidé de vivre leur vie en prenant tous les risques ${ }^{2}$. »

En tant que chaîne catalane publique, TV3 est doublement contrôlée par le CAC (Consell Audiovisual de Catalunya) et par la

1 « TV 3 aposta a partir de dijous que ve per una sèrie de dones, Infidels ", El Punt, 19/III/2009, p. 38. Je traduis, comme pour les autres citations de la suite de l'article.

${ }^{2}$ Ibid. 
Generalitat, le gouvernement autonome catalan ${ }^{3}:$ les programmes sont donc soumis à d'importantes exigences en matière de lutte contre les inégalités entre hommes et femmes. En effet, parmi les missions des médias publics catalans figure "la promotion active de l'égalité entre femmes et hommes, qui inclut l'égalité de traitement et d'opportunités, le respect de la diversité et de la différence, l'intégration de la perspective de genre, la promotion d'actions positives et l'emploi d'un langage non sexiste4, » comme le stipule la loi du 29 décembre 2005 régissant la communication audiovisuelle publique en Catalogne. On peut s'interroger sur les implications de ce code de conduite non-sexiste dans une fiction comme Infidels, revendiquée comme "une série dans laquelle les personnages masculins sont le contrepoint », où l'on a choisi de privilégier « un regard féminin sur le monde, et non un regard sur le monde féminin 5 . »

Il faut commencer par rappeler que l'autonomie des médias catalans face au pouvoir politique a justement été remise en question par les fidèles d'Infidels au moment où a été annoncée la fin de la série : certains y ont vu la trace d'une intervention des conservateurs de Convergència $i$ Unióo ${ }^{6}$, prétendument choqués par une série qualifiée d'«immorale7. » La rumeur a été vigoureusement démentie, tant par

3 «Créé en l'an 200o, le CAC est le reflet de la volonté des politiques nationales de communication de ces dernières années d'offrir aux médias une certaine indépendance visà-vis du pouvoir politique. Il s'agit d'un organisme régi par des membres proposés par le Parlement avec des mandats de six ans, afin de ne pas être lié excessivement aux législatures. Des mécanismes comme celui-ci ont visé à consolider les politiques nationales de communication contre les intérêts que pourrait avoir tel ou tel gouvernement. Ainsi, la Loi de la Corporation Catalane des Médias Audiovisuels (2007) donne également à TVC et Catalunya Ràdio une certaine indépendance vis-à-vis du gouvernement, même si la composition des instances dirigeantes de la Corporation reste largement politisée». Source : site web de la Generalitat,

http://www20.gencat.cat/portal/site/culturacatalana/[...], lien consulté le 17 juillet 2014. ${ }_{4}^{4}$ Llei 22/2005, de 29 de desembre, de la comunicació audiovisual de Catalunya, p. 24, http://www.cac.cat/pfw files/cma/normativa sa/Text consolidat Llei 22-2005.pdf, lien consulté le 17 juillet 2014.

${ }^{5}$ Déclaration du producteur exécutif Javier Olivares, « TV3 aposta a partir de dijous que ve per una sèrie de dones, Infidels ", op. cit.

6 Coalition des partis souverainistes de centre droit Convergència Democràtica de Catalunya et Unió Democràtica de Catalunya, souvent désignée par le sigle CiU.

7 Le quotidien Diari de Girona évoque ainsi la polémique : "La fin précipitée de la série Infidels, annoncée la semaine dernière par TV 3 [...], est à l'origine d'une forte polémique au sein du public. Nombreux sont les spectateurs qui considèrent que la fin de la série [...] répond à des motifs politiques et moraux en rapport avec l'arrivée de CiU au Gouvernement de la Generalitat. Le groupe Facebook « Nous ne voulons pas qu'on tue Infidels » est né, ainsi que le hashtag \#infidels sur Twitter, où de nombreux usagers dénoncent une censure. [...] Selon plusieurs médias en ligne, certains leaders du parti Unió Democràtica de Catalunya ont manifesté publiquement leur rejet de cette série en raison de son contenu 'immoral' ", "El punt i final de la sèrie Infidels continua aixecant polèmica ", Diari de Girona, 08/III/2011,

http://oci.diaridegirona.cat/tv/noticias/nws-9234-el-punt-i-final-serie-infidels-continuaaixecant-polemica.html, lien consulté le 17 juillet 2014. 
les responsables politiques que par les instances dirigeantes de la chaîne. Qu'ils soient fondés ou non, ces soupçons de censure méritent que l'on s'interroge: est-ce l'originalité revendiquée de la série, notamment en ce qui concerne la construction de ses protagonistes féminines, qui en fait un objet controversé ? Le cahier des charges antisexiste auquel sont soumis les programmes de TV 3 participe-t-il de cette originalité, et contribue-t-il, d'une manière ou d'une autre, à faire d'Infidels une série subversive?

Fait original, la réputation sulfureuse de la série a précédé son arrivée sur les écrans, et pas seulement à cause de son titre : en effet, TV3 a proposé au public une campagne de promotion constituée de cinq vidéos d'une trentaine de secondes chacune, diffusées quelques jours avant le premier épisode. Chaque teaser présente l'une des cinq protagonistes d'Infidels; plus exactement, chaque personnage se présente aux futurs téléspectateurs, en regardant la caméra et en prononçant une phrase qui évoque son rapport personnel à l'infidélité, fil conducteur apparent de la série. Penchons-nous, par exemple, sur la vidéo consacrée au personnage d'Arlet ${ }^{8}$, la benjamine. La phrase que prononce Arlet annonce qu'elle est en train de préparer la scène de l'infidélité qu'elle s'apprête à commettre : «Je n'ai jamais été infidèle. Jusqu'à maintenant ${ }^{9}$. " La musique qui accompagne chacune des vidéos, par son rythme lent et la voix de la chanteuse qui susurre plus qu'elle ne chante, achève de créer un horizon d'attente teinté d'érotisme ${ }^{10}$. Ces petites mises en bouche annoncent une série centrée sur la vie amoureuse et sexuelle de cinq femmes fictives que rien ne semble a priori distinguer des habitantes de Wisteria Lane ou de la bande de New-Yorkaises branchées gravitant autour de Carrie Bradshaw (voir figure 1).

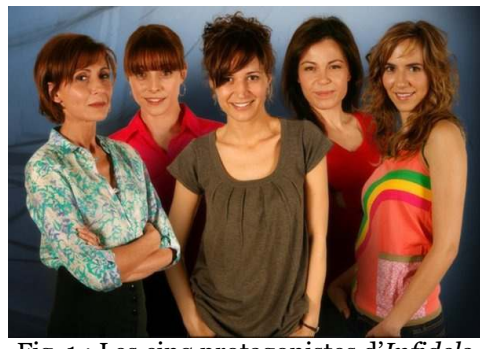

Fig. 1 : Les cinq protagonistes d'Infidels

${ }^{8} \mathrm{http}: / /$ www.youtube.com/watch?v=wMejUSOONNU, lien consulté le 18 juillet 2014.

9 Pour plus de commodité, toutes les répliques de personnages citées dans cet article ont été traduites en français.

${ }^{10}$ Il s'agit des premières mesures de la chanson « Sweet dreams (Are Made Of This) », composée et écrite par le groupe britannique Eurythmics (1983), reprise par la chanteuse québécoise Terez Montcalm. 
Cela nous amène à nous poser la question du cadre spatiotemporel, qui joue un rôle déterminant dans des séries telles que Desperate Housewives et Sex and the City. En effet, l'action de la première série ne se conçoit que dans une banlieue résidentielle étatsunienne dans la mesure où la fiction est centrée sur les "femmes au foyer » de la classe moyenne aisée. Quant au New York de Sex and the City, il s'agit en réalité de la recréation d'un microcosme, le petit monde de la communication, de la mode et de l'art contemporain dans lequel évoluent des personnages féminins culturellement, artistiquement et sexuellement avant-gardistes. En revanche, Infidels donne à voir des figures féminines qui esquissent un portrait assez contrasté de la classe moyenne barcelonaise. Le rôle symbolique du cadre apparaît comme limité dans la mesure où il semble avoir peu d'influence sur la construction des personnages qui le peuplent; tout du moins, il n'est pas lié à une unité entre les cinq protagonistes de la série, qui sont toutes issues de milieux différents (une institutrice, une gestionnaire de patrimoine, une psychiatre, une journaliste et une femme au foyer).

Pourtant, comme dans toutes les fictions catalanes, l'accent est mis sur la recréation d'un contexte extrafictionnel identifiable comme réel par les téléspectateurs : la ville de Barcelone est copieusement nommée et montrée, et l'ancrage de la fiction en Catalogne est régulièrement rappelé par la mention d'éléments de la réalité catalane actuelle, comme par exemple les groupes de musique qu'écoutent les personnages de la série ${ }^{11}$. C'est peut-être l'un des points communs les plus importants entre les cinq protagonistes d'Infidels : ces femmes d'âges et de milieux différents, que les hasards de la vie ont fait se rencontrer, partagent une langue et une culture qui non seulement s'imposent comme des évidences, mais qui jouent également un rôle non négligeable dans l'économie du récit. En effet, c'est grâce au comportement linguistique de son mari que Joana découvre qu'il mène une double vie : la fille de Joana, intriguée d'entendre son père parler en castillan au téléphone, ne le croit pas lorsqu'il lui dit qu'il parlait avec sa mère. Un peu plus tard, la fillette évoque les mensonges de son père auprès d'une des amies de sa mère :

MARINA. Je l'ai entendu parler au téléphone, et il a dit qu'il parlait avec maman.

ARLET. Et pourquoi est-ce que cela ne serait pas vrai ?

MARINA. Parce qu'il parlait en castillan, et qu'ils ne font jamais

ça. (1.2)

Face à l'argument imparable de la petite Marina, Arlet reste bouche bée : un couple catalan n'a aucune raison d'employer le castillan au

${ }^{11}$ Dans l'épisode 6 de la saison 1, Arlet offre à son fiancé Víctor des places pour un concert du groupe pop majorquin d'expression catalane Antònia Font. 
quotidien. C'est donc à cause de l'utilisation d'une langue plutôt que d'une autre que Joana finit par apprendre que son mari a fondé une autre famille à Buenos Aires, ville où ses affaires le retiennent une partie de l'année.

Parmi les protagonistes, seule Cruz s'exprime parfois en castillan, lorsqu'elle parle avec sa mère: la plus fortunée des cinq amies est la fille d'une femme de ménage d'origine andalouse. Cruz, à l'instar d'autres personnages de séries diffusées sur TV3, incarne le "rêve catalan ${ }^{12}$ »: elle est l'exemple d'une ascension sociale fulgurante allant de pair avec une irréprochable intégration culturelle et linguistique, dans la mesure où elle est presque exclusivement catalanophone. De fait, Cruz est l'épouse d'Eduard De Queralt, dont le patronyme ne laisse aucun doute quant à son appartenance à la vieille bourgeoisie catalane. Les repas de famille donnent d'ailleurs une curieuse impression: la mère de Cruz est la seule à s'exprimer en castillan, tandis que Cruz, Eduard et le père de celui-ci n'utilisent que le catalan, y compris lorsqu'ils s'adressent à elle (1.2). La visibilité du castillan est réduite au strict minimum dans la série, comme si le catalan était la seule langue parlée au quotidien, alors que la situation réelle est bien plus complexe. Ce traitement des langues dans les fictions de $\mathrm{TV}_{3}$ correspond à une stratégie de promotion de la langue catalane $^{13}$. Peter, le voisin de Joana, constitue un autre exemple de la volonté des concepteurs de la série de montrer le catalan comme la seule langue du quotidien : le jeune homme, originaire de Boston et récemment arrivé à Barcelone, parle un catalan presque parfait, à peine teinté d'un léger accent, et aucun des autres personnages de la série ne semble en être spécialement étonné.

La présence du personnage de Peter peut être vue comme une sorte de clin d'œil à un univers fictionnel ayant largement inspiré les créateurs d'Infidels, celui des séries états-uniennes. D'ailleurs, le fait que Joana, la maladroite de la bande, tombe sous le charme de son séduisant voisin (voir figure 2), ne peut qu'évoquer l'attirance de Susan Mayer pour Mike Delfino dans les premiers épisodes de Desperate Housewives.

\footnotetext{
${ }^{12}$ Pensons, par exemple, au personnage de Rafa, fils d'immigrés andalous, dans la série Ventdelplà. Cf. mon article "La série catalane Ventdelplà, entre réalisme et utopie ", TV/ séries, No. 2, 2012, p. 57-58 [p. 48-61].

${ }^{13}$ Selon la consultante linguistique d'une autre série de $\mathrm{TV}_{3}$, le traitement de la langue dans les fictions véhicule une sorte de "code de conduite " pour les catalanophones: "La fonction de la série avec les personnages d'immigrés est également de 'donner des pistes' aux gens sur la manière dont il faut agir linguistiquement face à des communautés qui ne parlent pas catalan [...]. Dans ce sens, on peut dire que la série de fiction intègre certains types de stratégies pour promouvoir le catalan ». Propos de Núria Comas, consultante linguistique de la série El cor de la ciutat, recueillis dans Enric Castelló, Bernat López, Identitat cultural $i$ societat a les séries de ficció catalanes : dels discursos a la recepció, Estudis de Comunicació de la URV, Tarragone, Universitat Rovira i Virgili, 2007, p. 33.
} 


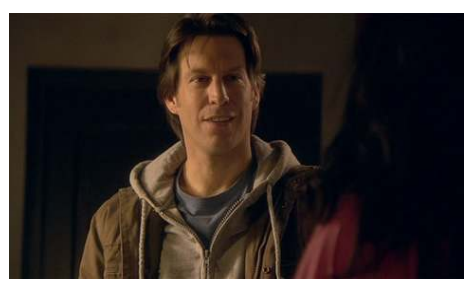

Fig. 2 : Peter, le voisin de Joana

À bien y regarder, les références plus ou moins évidentes aux fictions produites outre-Atlantique sont nombreuses et jouent sur la culture télévisuelle du spectateur, sur un ensemble de références partagé car faisant partie de l'encyclopédie du public contemporain. Lorsque la femme au foyer Joana quitte son mari infidèle et décide de subvenir à ses besoins et à ceux de ses enfants, Peter lui propose de l'aider à rédiger son CV. À la question : "Quel est ton profil ? ", elle répond : "Femme divorcée et désespérée » (1.7). On pourrait multiplier les exemples mais il semble plus intéressant de s'interroger sur la fonction de ces nombreuses citations, références et clins d'œil. L'apparente ressemblance entre Joana et Susan Mayer se révèle être, au fur et à mesure que la diégèse se déroule, une sorte de fausse piste : certes, ces deux femmes maladroites et gaffeuses ont également en commun de s'être retrouvées seules suite à l'infidélité de leurs époux respectifs et de tomber amoureuses de séduisants voisins, mais les similitudes s'arrêtent là. Tout au long de la série Desperate Housewives, le personnage campé par Teri Hatcher est une femme-enfant rêveuse, immature, dont la préoccupation première semble être le regard des autres, qu'il s'agisse de l'homme qu'elle souhaite séduire ou des femmes avec lesquelles elle passe son temps à rivaliser. En revanche, la comédienne Montse Germán incarne un personnage beaucoup plus " rond ${ }^{14}$, » à plus d'un égard. Certes, sa fixation sur ses rondeurs sert de prétexte à plusieurs séquences comiques : par exemple, dès le premier épisode, elle apparaît face à un miroir, s'efforçant d'enfiler un bustier. Son fils, hors champ, lui demande: «Maman, quand est-ce qu'on dîne ? » et elle répond, l'air désabusé : « Vous, tout de suite. Moi, dans trois jours. » (1.1) Cependant, lorsqu'elle découvre la double vie de son mari, Joana décide de le quitter et de reprendre les rênes de sa vie, après des années passées à s'occuper uniquement de sa maison et de ses enfants. C'est seulement bien après son divorce, une fois que Joana est indépendante, financièrement et émotionnellement, que commence sa relation avec son jeune voisin Peter: au lieu de se réfugier dans les bras d'un homme et de chercher à combler le vide

${ }_{14}$ Nous pensons à l'opposition entre flat characters et round characters établie par E.M. Forster dans Aspects of the Novel, Londres, Abinger edition, 1974 (1927). 
laissé par son ex-mari, Joana se bat pour acquérir une autonomie qu'elle refuse de remettre en question. Quand Peter lui propose de venir vivre avec elle, Joana hésite, puis décline son offre : "Je ne me vois pas partager les tâches ménagères avec toi, emmener les enfants à l'école ou repasser ton linge.» (1.16) La formation d'un nouveau couple n'est pas présentée comme le but à atteindre par le personnage féminin: la relation que Joana entretient avec Peter est un des éléments d'une nouvelle vie qu'elle s'est forgée à la force du poignet; elle participe de son bonheur et de son équilibre sans toutefois les conditionner totalement.

L'un des traits d'originalité d'Infidels est l'importance donnée à l'activité professionnelle de certaines des protagonistes:elle joue parfois un rôle fondamental dans l'économie du récit. C'est le cas pour Cruz, qui apparait très régulièrement dans son bureau, occupée à des tâches qui évoquent la complexité et la technicité de son métier de gestionnaire de patrimoine. C'est d'ailleurs sur le terrain de la finance que s'exprime sa rivalité avec la maîtresse de son mari, et non dans un domaine plus classique comme celui de la séduction. Lorsqu'elle découvre la liaison adultère d'Eduard avec Carlota, une riche héritière dont elle gère la fortune, Cruz se venge en investissant délibérément une partie de l'argent de sa rivale sur une opération qu'elle sait vouée à l'échec (1.4). Un peu plus tard, quand Eduard hérite de son père une agence immobilière criblée de dettes, Carlota lance à Cruz une sorte de provocation en duel : elle a les moyens d'aider Eduard à remettre à flot l'entreprise familiale et elle défie Cruz de pouvoir en faire autant. En bonne épouse aimante, tant pour sauver l'élu de son cœur que pour neutraliser une fois pour toutes son encombrante rivale, Cruz se livre à une opération financière aussi complexe qu'illégale (1.9). Alors qu'elle se rend ainsi coupable d'un délit d'initié et qu'elle met de côté l'éthique qui lui est pourtant chère, avec les risques que cela suppose pour sa carrière, Cruz s'inscrit dans une longue tradition de personnages féminins sacrificiels pour lesquels amour rime avec renoncement ${ }^{15}$. Ce qui est original ici, c'est la manière dont s'exprime l'abnégation de la femme amoureuse, à travers ses compétences professionnelles. Le personnage de Cruz est en cela à la fois désespérément classique et extrêmement original. La figure de la maîtresse d'Eduard met justement en valeur cette originalité, par contraste : Carlota, fille de bonne famille sans métier connu, n'apparaît qu'à travers sa relation

${ }^{15}$ Nous pourrions proposer de nombreux exemples, tant ces personnages sont nombreux dans les productions culturelles. Qu'il nous soit permis de faire ici une incursion dans le domaine de la musique et d'évoquer la chanson de country "Stand by your man " (Tammy Wynette et Billy Sherill, 1968), dont je reproduis ici un extrait qui, selon moi, parle de luimême : "Sometimes it's hard to be a woman,/giving all your love to just one man. /You'll have bad times and he'll have good times / doing things that you don't understand. / But if you love him you'll forgive him, /even though he's hard to understand./ And if you love him, /oh, be proud of him!/ Cause after all he's just a man./ Stand by your man, and show the world you love him. /Keep giving all the love you can ». 
avec Eduard, qu'elle côtoie depuis l'enfance et dont elle est amoureuse depuis presque aussi longtemps. Lorsqu'Eduard l'informe de son désir de mettre fin à leur liaison adultère, Carlota, au bord des larmes, plaide sa cause dans un monologue qui n'a rien à envier aux déclarations enflammées des personnages torturés de soap operas ou de culebrones (voire figure 3). Elle évoque la première fête à laquelle elle a participé, à l'âge de seize ans, dans la résidence familiale de Cadaqués, et l'éveil de ses sentiments pour Eduard: "Ce soir-là, je me suis promis que nous serions ensemble un jour. " (1.4) Contrairement aux cinq protagonistes d'Infidels, Carlota évolue dans un milieu social fermé qui apparaît comme isolé du monde réel, et elle semble n'avoir d'autre préoccupation dans l'existence que son apparence et ses déconvenues sentimentales : elle est sans doute à lire comme une sorte de transfuge venue d'autres univers fictionnels, clin d'œil aux feuilletons dont la télévision catalane a toujours eu à cœur de se démarquer, et contrepoint aux figures féminines originales que donne à voir la série.

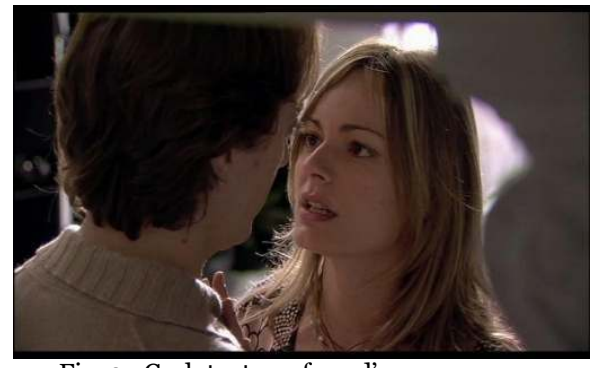

Fig. 3 : Carlota, transfuge d'un soap opera

Les protagonistes d'Infidels ont en commun un certain nombre de caractéristiques qui les opposent à la grande majorité des figures féminines qui peuplent les fictions télévisées. Il n'y a, dans la série, ni femme boniche, ni femme potiche ${ }^{16}$ : seule Joana, mère au foyer, est parfois montrée en train d'exécuter des tâches ménagères. C'est également la seule des protagonistes à exprimer explicitement une préoccupation pour son apparence, et cet élément est traité, comme nous l'avons vu, de manière plutôt comique. Une fois séparée de son mari, Joana se met à travailler ${ }^{17}$, n'apparaît plus que très sporadiquement avec une serpillière ou une casserole à la main et ne semble plus s'inquiéter de son surpoids imaginaire, comme si le fait de commencer à vivre pour elle-même et non plus pour sa famille avait radicalement bousculé ses préoccupations. Le rôle nourricier

${ }^{16} \mathrm{Cf}$. Claude Alzon, La Femme potiche et la femme boniche. Pouvoir bourgeois et pouvoir mâle, Paris, Maspéro, 1973.

17 Joana reprend également ses études dans la deuxième saison. 
traditionnel des femmes et son complément contemporain, celui d'objet esthétique et sexuel, sont remis en question par la série d'une manière qui mérite commentaire. De la même façon que la diégèse s'ancre dans un univers politique, culturel et linguistique idéal - une sorte de Catalogne utopique, qui ne parlerait que catalan -, elle a pour cadre une société dont le sexisme semble avoir été pratiquement éliminé, ou tout du moins être en voie d'extinction. Dans les différentes sphères professionnelles qui sont données à voir, le plafond de verre n'a pas l'air d'exister, les femmes ont les mêmes perspectives d'évolution de carrière que leurs homologues masculins et elles occupent souvent des postes à responsabilités. Quand Paula se voit proposer une promotion, elle dit à son mari Marc qu'elle pense la refuser car elle craint d'être trop accaparée par de nouvelles responsabilités qui risquent de la retarder dans l'écriture de son roman. Marc, journaliste comme Paula, reproche vivement à son épouse son manque d'ambition (1.1) : le relativisme de genre habituel est ici absent - Marc voit Paula comme une professionnelle, comme une égale censée se consacrer corps et âme à son travail et à la compétition pour atteindre le plus haut niveau de responsabilités et de prestige.

Lorsqu'elles sont évoquées, chose assez rare, les tâches domestiques sont équitablement réparties entre les conjoints. Après la rupture entre Cruz et Eduard (1.12), il suffit d'un seul plan pour que Joana et le téléspectateur découvrent que ce dernier s'est installé chez sa maîtresse : Joana, qui fait du porte-à-porte pour vendre des ustensiles de cuisine, se retrouve par hasard à proposer un de ses appareils miracles à Carlota. Alors que celle-ci tente de décliner l'offre, Eduard apparait dans le champ, derrière elle, portant un tablier : il est ainsi immédiatement identifié comme habitant de la maison et non comme simple visiteur (voir figure 4). Du même coup, l'association automatique cuisine-personnage féminin est implicitement remise en question, une fois de plus : le partage des tâches n'est pas l'exception mais la norme, du moins pour la génération des personnages principaux.

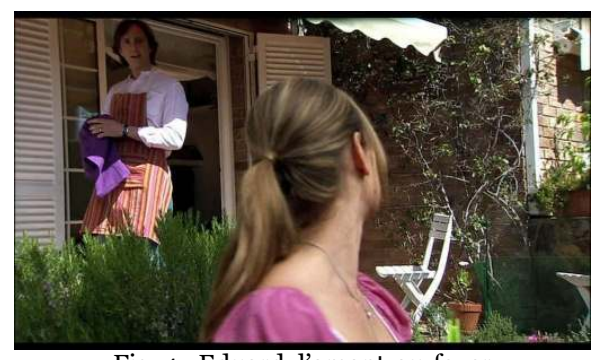

Fig. 4 : Eduard, l'amant-au-foyer 
En revanche, les choses sont bien différentes pour les personnages plus âgés, et le contraste suggère une considérable évolution de la condition féminine et des rôles de genre. Lorsque Cruz et Eduard annoncent à leurs parents qu'ils attendent un enfant, ces derniers se réjouissent et imaginent que la future mère va mettre un frein, sinon un terme, à sa brillante carrière. La mère de Cruz relativise l'importance du succès professionnel de sa fille face à la maternité, priorité absolue selon elle. Quant au père d'Eduard, il renchérit en disant qu' "une femme ne se réalise complètement que quand elle devient mère. » (1.2) De manière générale, la vision qu'ont les personnages âgés des rôles de genre relève du cliché : la mère de Cruz et le père d'Eduard sont des figures secondaires assez plates, dont la fonction essentielle semble être d'évoquer une évolution fulgurante de la condition des femmes catalanes en quelques décennies. En ce sens, on pourrait les rapprocher des parents d'Arlet, à travers lesquels est abordé le thème de la violence conjugale. Si le père d'Eduard est une sorte d'archétype de bourgeois catalan pétri de convictions surannées, celui d'Arlet concentre les traits caractéristiques les plus sombres de l'homme machiste: il exige d'être servi en toutes circonstances et maltraite sa femme. Lorsqu'il apprend que sa fille a rompu avec son fiancé, il lui reproche de ne pas avoir fait assez d'efforts pour le " garder », comme si la bonne marche d'une relation relevait de la responsabilité exclusive des femmes, et comme si la rupture ne pouvait avoir eu lieu qu'à l'initiative du partenaire masculin - comme si une femme, objet passif, ne pouvait qu'être abandonnée (1.11). Dans un mouvement d'humeur, Arlet finit par avouer à ses parents le véritable motif de la séparation : elle a quitté Víctor pour Dani, sa monitrice d'aquagym.

La relation entre Arlet et Dani se tisse progressivement, au fil des premiers épisodes, alors qu'Arlet est en couple avec un homme et que Dani collectionne les conquêtes masculines. Le trouble qui s'empare des deux jeunes femmes lorsqu'elles sont ensemble apparaît comme évident, mais elles mettent beaucoup de temps à reconnaître qu'elles sont attirées l'une par l'autre, jusqu'au moment où elles s'embrassent, timidement, à la fin du cinquième épisode. Un peu plus tard, Arlet invite Dani à dîner, mais celle-ci lui fait faux bond, visiblement mal à l'aise, comme le suggère l'explication qu'elle lui donne le lendemain : «Arlet, nous sommes amies et tu es géniale, mais je ne ressens rien de plus pour toi, le baiser de l'autre fois a été une erreur. [...] Je suis parfois un peu embrouillée, mais s'il y a une chose qui est très claire pour moi, c'est que je ne suis pas lesbienne ». Arlet lui répond: "Mais moi non plus!» (1.6) Les deux femmes nient l'attirance qu'elles ressentent l'une pour l'autre, car l'accepter les ferait entrer dans une catégorie à laquelle elles refusent d'appartenir. Arlet et Dani tardent beaucoup à accepter leurs sentiments et à officialiser leur 
relation, qui provoque des réactions contrastées auprès de leur entourage. Les amies d'Arlet ne semblent pas choquées, ni plus surprises que lorsque Joana leur présente son jeune amant américain. En revanche, l'attitude d'autres personnages mérite commentaire, à commencer par celle du père d'Arlet. Lorsqu'il rencontre Dani, il refuse de lui parler et lui dit à propos de sa fille : «Elle était normale avant que tu ne t'en mêles. » (1.11) Dani ne relève pas l'accusation implicite d'anormalité dont elle est l'objet mais réagit sur l'adjectif et renvoie le père d'Arlet à son propre comportement, en répliquant calmement : « Je n'arrive pas à comprendre votre conception de la normalité. Pour moi, il n'est pas très normal de foutre des baffes à sa famille. » (1.11) Le père d'Arlet étant caractérisé de manière négative dès sa première apparition, ce qui est rappelé ici à travers la mention de la violence qu'il exerce à l'encontre son épouse, son jugement apparaît comme totalement dépourvu de crédibilité: son rejet de l'homosexualité achève de le dépeindre comme une caricature d'homme machiste et intolérant; du même coup, associer l'homophobie à ce personnage, c'est la condamner sans appel, c'est la dénoncer comme un comportement primaire et agressif. L'attitude de Víctor face à la relation naissante entre son ex-petite amie et Dani mérite également commentaire car Víctor refuse de prendre acte de la rupture, considérant que les sentiments d'Arlet pour une femme ne peuvent être qu'une passade : "Arlet, ce qui t'arrive, c'est que tu es aveuglée par cette fille. [...] C'est temporaire. Tu veux expérimenter? D'accord, expérimente. [...] Elle t'a juste allumée, et quand ça te passera, je te manquerai » (1.10). Après le père d'Arlet, Víctor offre une seconde variation sur le thème de l'hétérosexualité obligatoire: une liaison entre deux femmes ne peut être qu'un caprice répondant à une pulsion passagère, une toquade insatisfaisante qui se terminera invariablement par un retour à l'homme, seul apte à combler les désirs et aspirations profondes d'une femme. Or, Víctor brille davantage par sa plastique que par son intelligence : là encore, le message est clair - Infidels évoque les idées reçues sur l'homosexualité (féminine, ici) et suggère qu'elles sont aussi stupides qu'infondées.

Cependant, Arlet et Dani s'interrogent également sur leur attirance et leur orientation amoureuse, n'ayant jamais connu, ni l'une ni l'autre, de relations autres qu'hétérosexuelle :

ARLET (à Dani). Tu te considères comme lesbienne ?

DANI. Je suis avec toi, non?

ARLET. Oui, mais on a toujours été avec des mecs.

DANI. Qu'est-ce que tu veux dire? Qu'on est bisexuelles?

ARLET. Mais pourquoi est-ce que les gens ont besoin de mettre

des étiquettes partout? (1.13) 


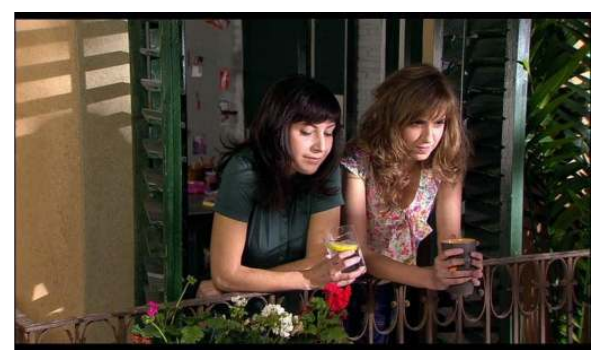

Fig. 5 : Deux amoureuses refusant l'étiquetage

C'est sur ces mots que se terminent le dialogue et la séquence: Arlet refuse de "s'étiqueter » pour satisfaire les autres, et refuse de faire de son désir une caractéristique définitoire. D’ailleurs, le couple formé par ces deux personnages remet en question l'étanchéité des catégories d'orientation sexuelle, et jusqu'à leur existence : ces deux femmes, qui jusqu'alors ne fréquentaient que des hommes, sont tombées amoureuses l'une de l'autre, mais ne changent pas pour autant d'identité en "devenant » lesbiennes. Comme le sexisme, l'homophobie et les idées reçues hétéronormatives sont cantonnées à des personnages caractérisés négativement et/ou représentant une époque révolue ; mais la série ouvre également des pistes de réflexion sur le désir, l'amour et l'orientation sexuelle, sur les multiples formes qu'ils peuvent prendre et sur l'inutilité, voire l'absurdité, des classements et des hiérarchisations dans ce domaine.

Infidels, comme l'immense majorité des programmes diffusés sur TV3, s'inscrit dans la continuité des fictions catalanes « maison », pensées et élaborées par des Catalans pour des Catalans, et peut être considérée à cet égard comme un produit méritant son " appellation d'origine contrôlé $\mathrm{e}^{18}$, » bien qu'ayant selon toute vraisemblance été inspiré par des recettes étrangères. Loin de se limiter à transposer à la réalité barcelonaise les séries états-uniennes à succès, les concepteurs d'Infidels ont construit tout un système de références ayant vraisemblablement un autre but que le simple plaisir du jeu et de l'identification des clins d'œil. En effet, la série semble citer ses apparents modèles pour mieux s'en démarquer; cela participe de la construction de figures féminines complexes et originales, et met en valeur leur dimension novatrice en rappelant celles qui les ont précédées sur les écrans. Certes, on pourrait reprocher à Infidels le manque de réalisme du cadre spatio-temporel dans lequel s'inscrit la diégèse : en plus de donner l'image d'une Catalogne exempte de ques-

${ }^{18}$ Cf. Joana Gallego, «Els serials catalans : un nou producte amb denominació d'origen », in Anàlisi, No. 23, 1999, p. 17-24. 
tionnements quant à l'identité nationale (caractéristique récurrente des fictions de TV3), la série donne à voir un univers utopique dont le sexisme et l'homophobie semblent avoir été presque totalement éliminés chez les nouvelles générations.

Cependant, en construisant une société fictionnelle libérée d'un certain nombre de problèmes «réels» d'inégalités et de discrimination, Infidels propose un questionnement qui aurait sans doute difficilement pu être abordé si la fiction s'était inscrite dans un cadre plus « réaliste », par exemple en ce qui concerne les assignations de genre, qui sont amplement questionnées. Les personnages masculins, que je me propose d'étudier dans un travail ultérieur ${ }^{19}$, sont particulièrement intéressants à cet égard. De plus, si la série fait l'impasse sur la répartition inégale des tâches ménagères ou sur les difficultés qu'ont les femmes dans le monde du travail, on peut certes y voir un traitement utopique de la condition féminine, mais il me semble qu'une autre interprétation est possible, si l'on établit un parallèle avec la question linguistique et culturelle : est-ce qu'il ne s'agit pas, tant pour les concepteurs du programme que pour les instances dirigeantes de la chaîne publique catalane, d'offrir aux téléspectateurs une représentation ayant valeur d'exemple?

\section{Bibliographie}

« TV3 aposta a partir de dijous que ve per una sèrie de dones, Infidels », El Punt, 19/III/2009, p. 38.

« El punt i final de la sèrie Infidels continua aixecant polèmica », Diari de Girona, 08/III/2011.

Alzon Claude, La Femme potiche et la femme boniche. Pouvoir bourgeois et pouvoir mâle, Paris, Maspéro, 1973.

CASTELlo Enric, LOPEZ Bernat, Identitat cultural i societat a les séries de ficció catalanes: dels discursos a la recepció, Estudis de Comunicació de la URV, Tarragone, Universitat Rovira i Virgili, 2007.

Forster Edward Morgan, Aspects of the Novel, Londres, Abinger edition, 1974 (1927).

GALLEGO Joana, «Els serials catalans: un nou producte amb denominació d'origen ", Anàlisi, No. 23, 1999, p. 17-24.

${ }^{19}$ Ce questionnement a donné lieu à un article intitulé «Confusion des genres et troubles dans les rôles : la série catalane Infidels ", qui sera prochainement publié aux Presses Universitaires de Limoges, dans la collection Féminin Masculin. 
HOUDIARD Jennifer, « La série catalane Ventdelplà, entre réalisme et utopie », TV/séries, No. 2, 2012, p. 48-61.

\section{L'auteure}

Jennifer Houdiard est Maître de conférences à l'université de Nantes. Ses recherches portent sur la prose narrative contemporaine espagnole et catalane, ainsi que sur la télévision catalane, et s'intéressent tout particulièrement à la thématique de l'identité (nationale et de genre) et de ses représentations dans la fiction. Elle est rattachée à titre principal au CRINI (Centre de Recherche sur les Identités Nationales et l'Interculturalité, Université de Nantes), et au GRIC (Groupe de Recherche Identités et Cultures, Université du Havre) en tant que membre associé. 